\title{
Maternal Health in the Transgender Population
}

\author{
Sajal Patel, MD, and Lori B. Sweeney, MD²
}

\begin{abstract}
Social acceptance and legal protections for transgender and gender nonconforming patients have increased over the past decade, but significant health care disparities still remain. Such an area of disparity is discussion with and interventions for fertility, contraception, pregnancy, and lactation in TGNC patients. Providing optimal care starts with creating a welcoming and safe environment. Appropriate preconception education includes the effects of gender-affirming therapies (both surgical and nonsurgical) on fertility as well the fertility preservation techniques that are available. However, as gamete retrieval requires natal hormone stimulation, gender dysphoria may be worsened. Thus, these patients should be carefully monitored not only medically, but also with regard to their mental health. In addition to assisted reproductive technologies, protocols exist to aid with induction of lactation as well as discontinuation if desired. As this is a growing field of medicine with limited data available on safety and long-term outcomes, recommendations are for a multidisciplinary team approach to ensure patients' safety and well-being.
\end{abstract}

Keywords: transgender, reproductive, fertility

\section{Introduction}

C URRENT estimates SUgGeST that $0.6 \%$ of the American population identifies as transgender or gender nonconforming transgender or gender nonconforming (TGNC). ${ }^{1} \mathrm{~A}$ TGNC person is one whose gender identity differs from that which was assigned at birth. ${ }^{2}$ If an individual's gender identity does not fit within the male/female dichotomy, the description "nonbinary gender identity" can be applied. ${ }^{3}$ Gender dysphoria is defined as the distress experienced when gender identity and designated gender are not completely congruent. ${ }^{2}$ A TGNC person may choose to "transition" or change their physical, social, and legal characteristics to align with the identified gender. ${ }^{2}$ TGNC patients often face discrimination and heteronormative attitudes when seeking health care, which may lead to health disparity and negative health outcomes. Fortunately, over the past decade, social acceptance and legal protections have increased for this population. In addition, many providers have gained competency in the medical care of TGNC patients, including the provision of gender-affirming therapies. However, therapies such as hormone administration and gender-reassignment surgery may adversely affect reproductive potential. ${ }^{4}$ The care of these patients should, therefore, address potential issues that span the reproductive continuum, and reproductive goals should be discussed early. This article examines fertility, contraception, pregnancy, and lactation in TGNC patients and provides a framework for reproductive planning relative to the patient's history of gender-affirming therapies.

\section{The Affirming Clinical Environment}

The first step in addressing the reproductive health care needs of the TGNC patient is creating an affirming clinical environment. Transgender care requires providers to anticipate and address the unique needs of the TGNC population. All personnel who will engage with TGNC patients should receive adequate training on transgender health issues and the use of inclusive language. Waiting rooms can be made more welcoming through the designation of gender-neutral bathrooms and addition of LGBTQ themed visual cues (pamphlets, posters, and artwork). ${ }^{5}$ Asking patients about their preferred pronouns and preferred terms for referencing parts of the anatomy is important. TGNC patients may elect

\footnotetext{
${ }^{1}$ Division of Endocrinology, Diabetes, and Metabolism, Virginia Commonwealth University Hospital System, Richmond, Virginia, USA. ${ }^{2}$ Division of Endocrinology \& Metabolism, Central Virginia VA Health Care System, Richmond, Virginia, USA.

(c) Sajal Patel and Lori B. Sweeney 2021; Published by Mary Ann Liebert, Inc. This Open Access article is distributed under the terms of the Creative Commons Attribution Noncommercial License (http://creativecommons.org/licenses/by-nc/4.0/) which permits any noncommercial use, distribution, and reproduction in any medium, provided the original author(s) and the source are cited.

Correction added on February 19, 2021 after first online publication of December 4, 2020: The article reflects Open Access, with copyright transferring to the author(s), and a Creative Commons Attribution Noncommercial License (CC-BY-NC) added (http:// creativecommons.org/licenses/by-nc/4.0/).
} 
for gender-affirming hormone therapy and/or surgery, which may affect secondary sexual characteristics and outward presentation. One potentially helpful practice is to have patients complete a self-inventory of anatomical parts, which can be reviewed at each clinical encounter. ${ }^{6}$ TGNC patients (especially transgender males [TGMs]) may prefer terms such as "chest" and "front hole" over more traditional terms such as "breast" or "vagina." 6,7 Providers also should bear in mind that terms such as "mother" and "father" may worsen gender dysphoria, and the term "parent" may be substituted.

Special considerations also exist for providers who will conduct an examination of the reproductive organs of TGNC patients. Physical touch by a provider may be particularly anxiety provoking for these patients. ${ }^{6}$ As such, providers should approach the physical examination and invasive procedures with this knowledge. Although the presence of a chaperone is routine, the role of each person in the examination room should be discussed with the patient. TGMs who are receiving testosterone therapy may develop vaginal and cervical atrophy, which can make a speculum examination more uncomfortable. Pretreatment of these patients with 2 weeks of vaginal estradiol may alleviate this and should not result in significant systemic absorption. ${ }^{6}$ The University of California, San Francisco Center of Excellence for Transgender Health, provides other recommendations for patients who are fearful of undergoing a pelvic examination. These include allowing the patient to have a support person in the room or the use of distracting techniques, such as listening to music with headphones. ${ }^{6}$ Utilization of a traumabased approach may be necessary, and pretreatment with oral benzodiazepine can be considered. ${ }^{6}$

\section{Gender-Affirming Therapies and Preconception}

Although gender-reaffirming surgeries, such as oophorectomy and orchiectomy, result in sterility, hormonal-based regimens may have permanent or reversible effects on reproductive potential. ${ }^{8}$ Transgender females (TGFs) may undergo therapy with estrogens, androgen blockers (spironolactone or cyproterone acetate), gonadotrophin-releasing hormonem (GnRH) agonists, and progestins. These therapies generally result in downregulation of gonadotropins and have variable effects on testicular structure and function. ${ }^{9,10} \mathrm{Im}$ pairments in spermatogenesis may occur as a result of hormone therapy, but examination of orchiectomy specimens has revealed inconsistent findings, and normal spermatogenesis may be present in some patients. ${ }^{11}$ TGMs may elect for treatment with testosterone, GnRH agonists, or progestins. GnRH agonists and progestins generally have been administered early in transition to achieve more rapid cessation of menses. These therapies can suppress ovulation through multiple mechanisms. Testosterone therapy may inhibit gonadotropin release and promote changes in the ovary that are similar to those changes that have been observed in cisfemales with androgen excess disorders such as polycystic ovary syndrome. ${ }^{9,12}$ Much of the available data regarding the potential adverse effects of gender-affirming hormones on fertility are conflicting. This highlights the importance of conducting discussions about reproductive goals before initiating treatment for gender dysphoria. Several options exist for TGNC patients who wish to have biologically related offspring. Table 1 summarizes available interventions.
TGMs may taper testosterone therapy, and spontaneous menses may resume within 6 to 12 months. ${ }^{19}$ If ovulation is restored, these patients can become pregnant with sexual intercourse, insemination, or assisted reproductive technologies (ARTs). ${ }^{15,19}$ TGMs may also undergo cryopreservation of oocytes, which can be used later by the patient, a female partner, or a gestational carrier. ${ }^{20}$ Likewise, TGFs may undergo cryopreservation of sperm to preserve fertility. ${ }^{9}$ In both cases, gamete retrieval requires hormonal stimulation, which may worsen gender dysphoria. Potential future options also exist for fertility preservation in prepubertal TGNC patients before starting gender-reaffirming hormone therapy. These methods are experimental and include ovarian or testicular tissue cryopreservation. Investigational protocols exist to promote proliferation and maturation of these immature gametes for the purposes of achieving fertility. ${ }^{21}$

\section{Fertility and the TGNC Patient}

Fertility preservation rates are low in TGNC patients. Published reports suggest the rates may be as low as $5 \%$ to $15 \%$ in adults and $2.8 \%$ in adolescents. ${ }^{22,23}$ This is in direct contrast to the $24 \%$ to $58 \%$ of TGNC patients who report the desire to have children. ${ }^{22}$ This may reflect the preference of TGNC patients to adopt, foster children, or pursue alternative family-building paradigms. ${ }^{23}$ However, studies have shown that the low utilization of fertility preservation may be due to a lack of knowledge on the part of the provider or patient regarding available options. ${ }^{23}$ In addition, TGNC patients may have concerns over the invasiveness of fertility treatments and the risk for worsening of gender dysphoria with discontinuation of hormone therapy. ${ }^{24}$ The cost of fertility preservation or ART also may represent an obstacle to utilization. Cost of sperm preservation ranges from $\$ 400$ to $\$ 1,000$ for collection and $\$ 200$ to $\$ 500$ per year for storage, and the cost of oocyte preservation ranges from $\$ 5,000$ to $\$ 20,000$ for collection (not including the cost for medication used to induce ovulation) and $\$ 100$ to $\$ 1,000$ per year for storage. ${ }^{13}$ Despite the aforementioned potential barriers, one study suggests $51 \%$ of transgender women and $38 \%$ of transgender men would have considered gamete cryopreservation if it had been offered before hormone initiation. ${ }^{9}$ Also, up to $20 \%$ of respondents to one survey had considered fertility preservation, but did not discuss it with a health care provider. ${ }^{25}$

\section{Contraception and the TGNC Patient}

It is important to recognize that TGMs may have unplanned pregnancies while on testosterone therapy even when amenorrhea is present. ${ }^{19}$ In addition, although estradiol therapy may inhibit spermatogenesis, a TGF could plausibly inseminate a female partner if viable sperm still are produced. ${ }^{18}$ As such, professional organizations recommend that all transgender people who retain their gonads and engage in sexual activity that could result in pregnancy be counseled on the need for contraception. ${ }^{15}$ Contraceptive therapies are particularly important in TGMs because testosterone and antiandrogen medications are teratogenic. It is well known that testosterone does not suffice as contraception because it does not reliably prevent ovulation. Unfortunately, a large portion of the transgender community believes that testosterone use represents a contraceptive measure. ${ }^{3}$ In a cohort of 


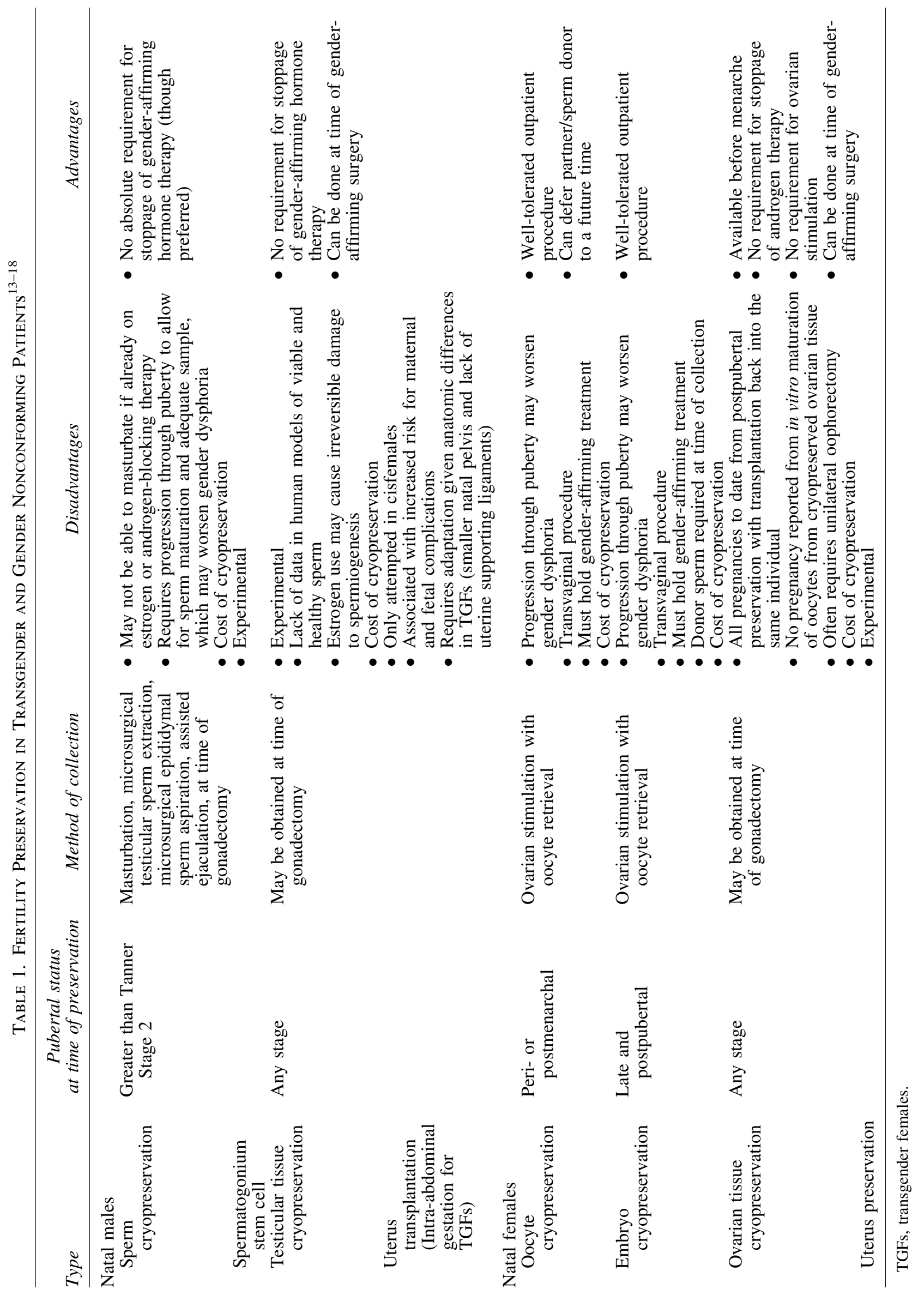




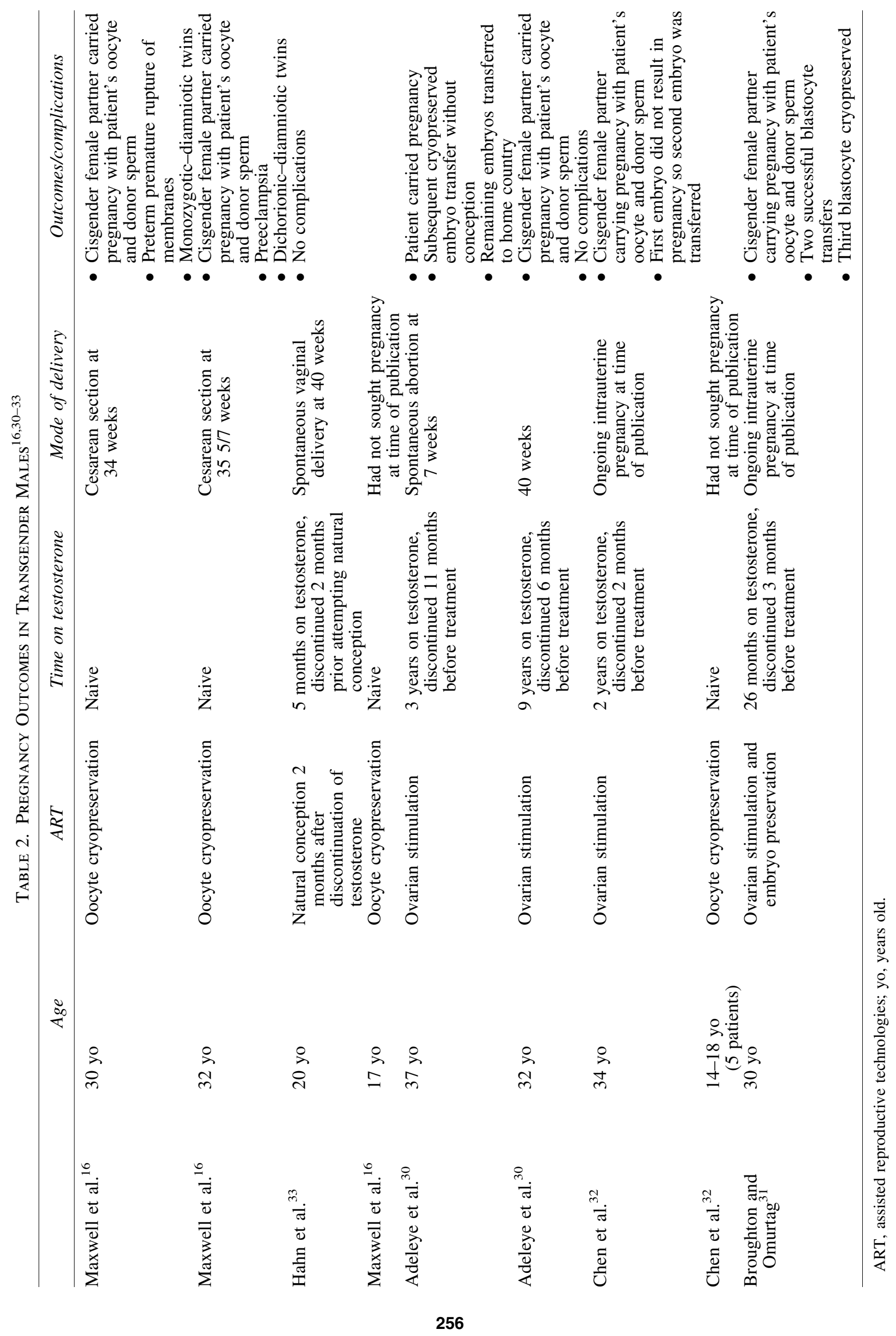


197 transgender men, $16 \%$ used testosterone as contraception, and $5.5 \%$ said health care providers had advised them this was a reliable method. ${ }^{26}$

The total number of pregnancies in transgender men is unknown, but studies suggest that pregnancy rates range from $5 \%$ to $17 \%$ in adolescents and adults. ${ }^{26,27}$ One study showed that TGNC youth had an unplanned pregnancy rate of $26 \%$ compared with $12 \%$ in their cisgender counterparts, and another study reported a rate of $40 \% .^{12,28}$ However, a study by Light et al. showed a $60 \%$ rate of using or having used contraception, which was similar to the national average of $62 \% .^{26}$ The misinformation about testosterone qualifying as a contraceptive measure, along with increased higher risk sexual behavior among TGNC patients, may contribute to the prevalence of unplanned pregnancies in this population. Additional considerations exist with regard to specific types of contraception in TGNC patients. Although TGM patients may prefer an IUD to other forms of contraception, the insertion may trigger dysphoria, and conscious sedation can be considered. Interestingly, transgender men who had been currently or formerly prescribed testosterone were more likely to use IUDs than those who were testosterone naive. ${ }^{26}$ In addition, as the prevalence of HIV approaches $19 \%$ in TGFs, condom use may be preferable as a mode of contraception given its efficacy in prevention of sexually transmitted infections. ${ }^{29}$

\section{Pregnancy and the TGNC Patient}

Data are limited regarding pregnancy outcomes and obstetric complications in TGM. Table 2 summarizes information derived from recent studies. Research extrapolated from cisgender females suggests an association between elevated endogenous androgen levels and reduced birth weight. ${ }^{34}$ Light et al. conducted a study of eight TGM parents to assess their experiences with conception, pregnancy, and birth. The participants self-reported preterm labor (10\%), placental abruption (10\%), anemia (7\%), and hypertension (12\%). Respondents included both users and nonusers of testosterone before pregnancy, and no trend was found in the proportion of complications relative to this history. Another finding of this study was an increased preference for elective cesarean as the mode of delivery among TGMs who had previously used testosterone. ${ }^{19}$ This mode of delivery may lessen dysphoriawhich can be triggered by a vaginal delivery - and should be discussed with patients. For surveillance of pregnancy, transabdominal ultrasound may be preferred over transvaginal ultrasound and can be undertaken when appropriate.

Although masculinizing hormone therapies must be stopped during pregnancy, providers should be aware that TGMs may experience significant mood swings, worsening of gender dysphoria, or worsening of underlying mental health disorders secondary to regression of masculinization and the appearance of feminine features that accompany pregnancy. This can occur even in TGMs who desire and plan for pregnancy. For some patients, masculinized features, such as facial hair, persist despite cessation of testosterone during pregnancy. Given the societal expectations regarding gender norms, a patient with mixed phenotypic characteristics may be more vulnerable to harassment and discrimination. TGMs may also control their gender presentation by dressing as an overweight male or binding their breasts. ${ }^{35}$ The use of a breast binder during pregnancy could interfere with lactation and increase the risk for complications such as mastitis. Several of these factors may make TGNC patients more likely to choose alternative settings for delivery of their babies and may influence their preferences for midwives or nonphysician providers. ${ }^{36}$

\section{Lactation and the TGNC Patient}

TGMs who gestate may wish to chestfeed. Providers should be supportive of the patient's decision in this regard. Those who pursue chestfeeding may encounter trouble with infant latch if they have previously undergone masculinizing chest surgery. Different surgical approaches result in variable degrees of mammary gland/milk duct preservation. ${ }^{7}$ TGMs should receive presurgical counseling on the potential impact of each technique relative to their future capacity to chestfeed. TGMs who would like to chestfeed without gestation can achieve lactation through the same hormone protocols used for cisgender women. In one case report, a TGF was administered estrogen, progesterone, and the galactagogue domperidone to achieve lactation. She also used a breast pump to stimulate secretion of oxytocin and prolactin. The patient was able to exclusively chestfeed her infant for the first 6 weeks of life, followed by inclusive feeding with formula thereafter. ${ }^{37}$ Unfortunately, domperidone is not approved by the U.S. Food and Drug Administration (FDA) because of risk of arrhythmias, cardiac arrest, and sudden death. The FDA also recommends against its use off-label due to unknown risks to the infant, but it is available in Canada for those who can obtain it. ${ }^{37}$ Metoclopramide also has been used off-label as a galactagogue, but is not FDA recommended because of its high secretion into breast milk. ${ }^{38}$

Because gender dysphoria may worsen with chestfeeding, some patients may desire to resume gender-affirming hormone therapy while continuing chestfeeding. The antiandrogen spironolactone has a low secretion rate into breast milk $(0.02 \%$ of the parental daily dose) and is potentially safe for the infant. ${ }^{39}$ Fewer rigorous data are available regarding the safety of resuming testosterone during lactation. Testosterone is known to suppress lactation, but studies show that because of its low oral bioavailability and limited secretion into breast milk, it is not likely to affect the baby adversely. ${ }^{40}$ Therefore, it is recommended that testosterone be discontinued during lactation induction, but it can be resumed if necessary once an adequate milk supply has been established. ${ }^{39}$

For those patients who wish to stop chestfeeding, $\mathrm{D}_{2}$ receptor antagonists may be used to suppress lactation, with cabergoline the better tolerated within this class. Bromocriptine is not FDA approved for this indication because of its association with maternal deaths from myocardial infarction. ${ }^{41}$ Nonmedication options include pumping only to relieve discomfort and using cold compresses for pain and inflammation. Excessive pumping or chest stimulation may result in increased milk production, so the wearing of clothing tight enough to prevent breast expansion is recommended, but not so much to cause mastitis. ${ }^{35}$

\section{Conclusions}

The increased societal acceptance of transgenderism or gender nonconformity will likely result in a greater proportion of TGNC young patients seeking medical care, which will allow medical providers to better address their reproductive needs. Providing these patients with up-to-date information on the fertility implications of gender-affirming 
therapies and the available options for fertility preservation, pregnancy, and lactation is critical. These conversations should commence before medical or surgical transition occurs. Providers also should recognize that fertility treatments - which necessitate stoppage of gender-affirming hormone therapy-pregnancy, delivery, and lactation may worsen gender dysphoria in TGNC patients. Furthermore, TGNC patients who become pregnant should be monitored for postpartum depression. ${ }^{36}$ As such, the care of TGNC patients is best accomplished with a multidisciplinary treatment team, including both medical and mental health providers.

\section{Author Disclosure Statement}

No competing financial interests exist.

\section{Funding Information}

No funding was received for this article.

\section{References}

1. Flores AR, Herman JL, Gates GJ, Brown TNT. How many adults identify as transgender in the United States? Williams Institute, 2016. Available at: https://williamsinsti tute.law.ucla.edu/wp-content/uploads/Trans-Adults-US-Aug2016.pdf Accessed March 1, 2020.

2. Hembree WC, Cohen-Kettenis PT, Gooren L, et al. Endocrine treatment of gender-dysphoric/gender-incongruent person: An Endocrine Society Clinical Practice guideline [published correction appears in J Clin Endocrinol Metab 2018;103(2):699)] [published correction appears in J Clin Endocrinol Metab 2018;103(7):2758-2759]. J Clin Endocrinol Metab 2017;102:3869-3903

3. Feigerlová E, Pascal V, Ganne-Devonec MO, Klein M, Guerci B. Fertility desires and reproductive needs of transgender people: Challenges and considerations for clinical practice. Clin Endocrinol (Oxf) 2019;91:10-21.

4. Goldman RH, Kaser DJ, Missmer SA, et al. Fertility treatment for the transgender community: A public opinion study. J Assist Reprod Genet 2017;34:1457-1467.

5. Deutsch, MB. Creating a safe and welcoming clinic environment. UCSF Transgender Care \& Treatment Guidelines. UCSF Center of Excellence for Transgender Health, 2016. Available at: https://transcare.ucsf.edu/guidelines/clinicenvironment/ Accessed March 4, 2020.

6. Wesp L. Transgender patients and the physical examination. UCSF Transgender Care \& Treatment Guidelines. UCSF Center of Excellence for Transgender Health, 2016. Available at: https://transcare.ucsf.edu/guidelines/physicalexamination/ Accessed March 7, 2020.

7. MacDonald T, Noel-Weiss J, West D, et al. Transmasculine individuals' experiences with lactation, chestfeeding, and gender identity: A qualitative study. BMC Pregnancy Childb 2016;16:106.

8. Neblett MF 2nd, Hipp HS. Fertility considerations in transgender persons. Endocrinol Metab Clin North Am 2019;48:391-402.

9. Moravek MB. Fertility preservation options for transgender and gender-nonconforming individuals. Curr Opin Obstet Gynecol 2019;31:170-176.

10. Schneider F, Kliesch S, Schlatt S, Neuhaus N. Andrology of male-to-female transsexuals: Influence of cross-sex hormone therapy on testicular function. Andrology 2017;5: 873-880.
11. Jiang DD, Swenson E, Mason M, et al. Effects of estrogen on spermatogenesis in transgender women. Urology 2019; 132:117-122.

12. De Roo C, Lierman S, Tilleman $K$, et al. Ovarian tissue cryopreservation in female-to-male transgender people: Insights into ovarian histology and physiology after prolonged androgen treatment. Reprod Biomed Online 2017; 34:557-566.

13. Nahata L, Chen D, Moravek MB, et al. Understudied and underreported: Fertility issues in transgender youth-A narrative review. J Pediatr 2019;205:265-271.

14. Cheng PJ, Pastuszak AW, Myers JB, Goodwin IA, Hotaling JM. Fertility concerns of the transgender patient. Transl Androl Urol 2019;8:209-218.

15. Mattawanon N, Spencer JB, Schirmer DA 3rd, Tangpricha V. Fertility preservation options in transgender people: A review. Rev Endocr Metab Disord 2018;19:231-242.

16. Maxwell S, Noyes N, Keefe D, Berkeley A, Goldman K. Pregnancy outcomes after fertility preservation in transgender men. Obstet Gynecol 2017;129:1031-1034.

17. Sterling J, Garcia MM. Fertility preservation options for transgender individuals. Transl Androl Urol 2020;9(Suppl 2): S215-S226.

18. Schneider F, Scheffer B, Dabel J, et al. Options for fertility treatments for trans women in Germany. J Clin Med 2019; $8: 730$.

19. Light AD, Obedin-Maliver J, Sevelius JM, Kerns JL. Transgender men who experienced pregnancy after femaleto-male gender transitioning. Obstet Gynecol 2014;124: $1120-1127$.

20. Amato P. Fertility options for transgender persons. UCSF Transgender Care \& Treatment Guidelines. UCSF Center of Excellence for Transgender Health, 2016. Available at: https://ranscare.ucsf.edu/guidelines/fertility/ Accessed February 28, 2020.

21. Martinez F. Update on fertility preservation from the Barcelona International Society for Fertility PreservationESHRE-ASRM 2015 expert meeting: Indications, results and future perspectives. Hum Reprod 2017;32:1802-1811.

22. Iwamoto SJ, Defreyne J, Rothman MS, et al. Health considerations for transgender women and remaining unknowns: A narrative review. Ther Adv Endocrinol Metab 2019;10:2042018819871166.

23. Tishelman AC, Sutter ME, Chen D, et al. Health care provider perceptions of fertility preservation barriers and challenges with transgender patients and families: Qualitative responses to an international survey. J Assist Reprod Genet 2019;36:579-588.

24. Chiniara LN, Viner C, Palmert M, Bonifacio H. Perspectives on fertility preservation and parenthood among transgender youth and their parents. Arch Dis Child 2019; 104:739-744.

25. Wierckx K, Van Caenegem E, Pennings G, et al. Reproductive wish in transsexual men. Hum Reprod 2012;27:483-487.

26. Light A, Wang LF, Zeymo A, Gomez-Lobo V. Family planning and contraception use in transgender men. Contraception 2018;98:266-269.

27. Veale J, Watson RJ, Adjei J, Saewyc E. Prevalence of pregnancy involvement among Canadian transgender youth and its relation to mental health, sexual health, and gender identity. Int J Transgend 2016;17:107-113.

28. Nahata L, Chen D, Quinn GP, et al. Reproductive attitudes and behaviors among transgender/nonbinary adolescents. J Adolesc Health 2020;66:372-374. 
29. HIV/AIDS: Transgender people. World Health Organization, 2020. Available at: https://www.who.int/hiv/topics/ transgender/about/en/ Accessed March 6, 2020.

30. Adeleye AJ, Cedars MI, Smith J, Mok-Lin. Ovarian stimulation for fertility preservation or family building in a cohort of transgender men. J Assist Reprod Genet 2019;36: 2155-2161.

31. Broughton D, Omurtag K. Care of the transgender or gender-nonconforming patient undergoing in vitro fertilization. Int J Transgend 2017;18:372-375.

32. Chen D, Bernardi LA, Pavone ME, Feinberg EC, Moravek MB. Oocyte cryopreservation among transmasculine youth: A case series. J Assist Reprod Genet 2018;35: 2057-2061.

33. Hahn M, Sheran N, Weber S, Cohan D, Obedin-Maliver J. Providing patient-centered perinatal care for transgender men and gender-diverse individuals: A collaborative multidisciplinary team approach. Obstet Gynecol 2019;134:959-963.

34. Cho J, Su X, Phillips V, Holditch-Davis D. Association of maternal and infant salivary testosterone and cortisol and infant gender with mother-infant interaction in very-lowbirthweight infants. Res Nurs Health 2015;38:357-368.

35. García-Acosta JM, San Juan-Valdivia RM, FernándezMartínez AD, Lorenzo-Rocha ND, Castro-Peraza ME. Trans* pregnancy and lactation: A literature review from a nursing perspective. Int J Environ Res Public Health 2019;17:44.
36. Obedin-Maliver J, Makadon HJ. Transgender men and pregnancy. Obstet Med 2016;9:4-8.

37. Reisman T, Goldstein Z. Case report: Induced lactation in a transgender woman. Transgend Health 2018;3:24-26.

38. Zuppa AA, Sindico P, Orchi C, Carducci C, Cardiello V, Romagnoli C. Safety and efficacy of galactogogues: Substances that induce, maintain and increase breast milk production. J Pharm Sci 2010;13:162-174.

39. MacDonald TK. Lactation care for transgender and nonbinary patients: Empowering clients and avoiding aversives. J Hum Lact 2019;35:223-226.

40. Glaser RL, Newman M, Parsons M, Zava D, GlaserGarbrick D. Safety of maternal testosterone therapy during breast feeding. Int J Pharm Compd 2009;13:314-317.

41. McGuire TM. Drugs affecting milk supply during lactation. Aust Prescr 2018;41:7-9.

Address correspondence to: Sajal Patel, $M D$

Division of Endocrinology, Diabetes, and Metabolism Virginia Commonwealth University Hospital System Richmond, VA 23298

USA

E-mail: sajalpate19@gmail.com 